SCIENCE, SOCIETY AND NEW TECHNOLOGIES SERIES RESEARCH FOR INNOVATIVE TRANSPORTS SET
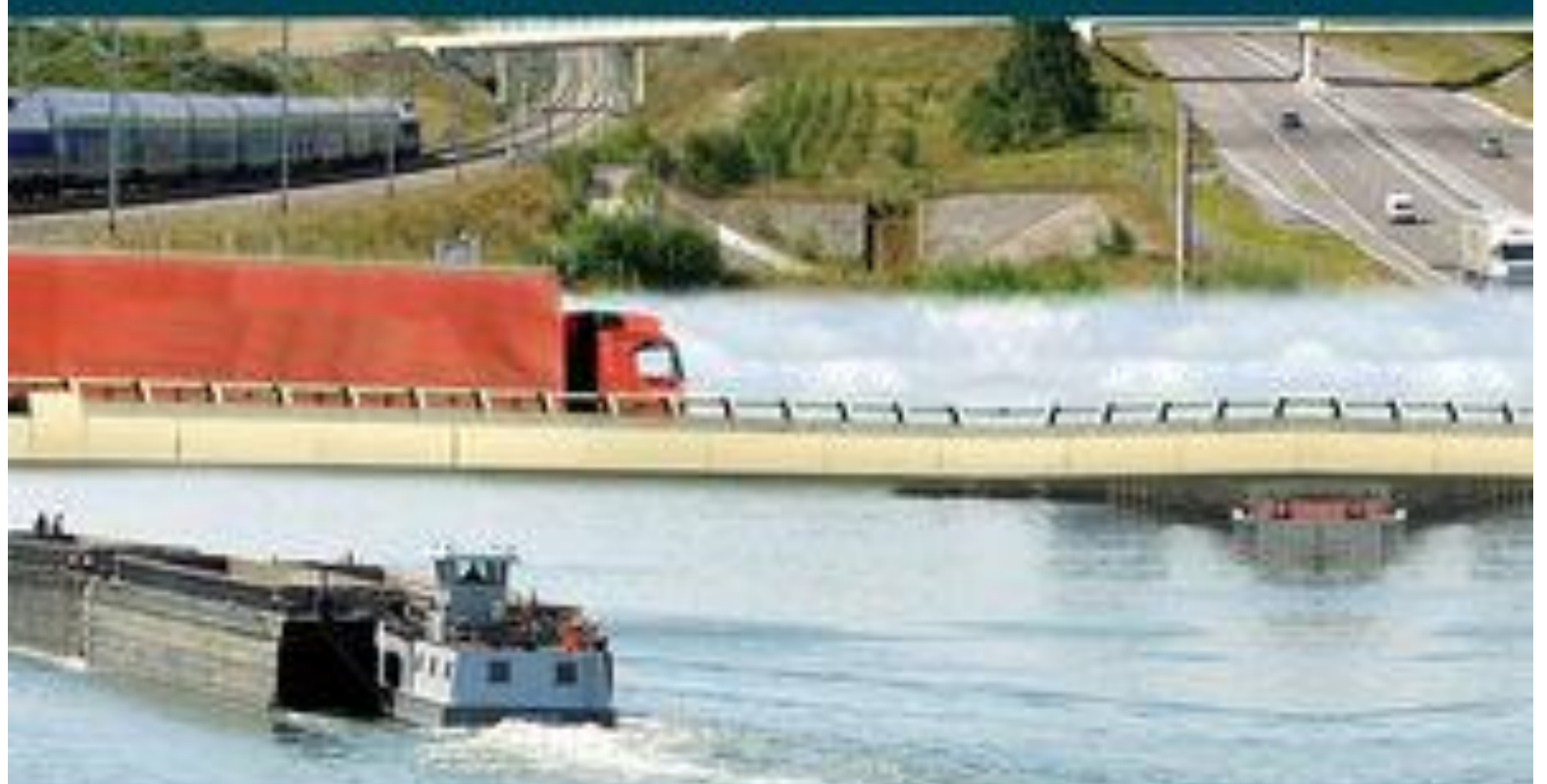

\title{
Volume 5A
}

\section{Materials and \\ Infrastructures 1}

\author{
Edited by
}

Jean-Michel Torrenti

Francesca La Torre

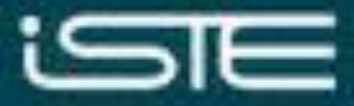

WILEY 
Materials and Infrastructures 1 
Research for Innovative Transports Set

coordinated by

Bernard Jacob

Volume 5A

\section{Materials and Infrastructures 1}

Edited by

Jean-Michel Torrenti Francesca La Torre

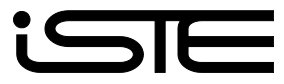


First published 2016 in Great Britain and the United States by ISTE Ltd and John Wiley \& Sons, Inc.

Apart from any fair dealing for the purposes of research or private study, or criticism or review, as permitted under the Copyright, Designs and Patents Act 1988, this publication may only be reproduced, stored or transmitted, in any form or by any means, with the prior permission in writing of the publishers, or in the case of reprographic reproduction in accordance with the terms and licenses issued by the CLA. Enquiries concerning reproduction outside these terms should be sent to the publishers at the undermentioned address:

\section{ISTE Ltd}

27-37 St George's Road

London SW19 4EU

UK

Www.iste.co.uk
John Wiley \& Sons, Inc.

111 River Street

Hoboken, NJ 07030

USA

www.wiley.com

\section{(C) ISTE Ltd 2016}

The rights of Jean-Michel Torrenti and Francesca La Torre to be identified as the authors of this work have been asserted by them in accordance with the Copyright, Designs and Patents Act 1988.

Library of Congress Control Number: 2016939896

British Library Cataloguing-in-Publication Data

A CIP record for this book is available from the British Library

ISBN 978-1-78630-029-4 


\section{Contents}

Preface

xix

Acknowledgments $\ldots \ldots \ldots \ldots \ldots \ldots \ldots \ldots \ldots \ldots \ldots$ xiii

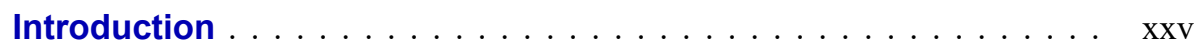

Part 1. Materials for Infrastructures. . . . . . . . . . . . . 1

Chapter 1. Use of an Ultra-wide Band

Radar to Detect Slope Movements Along

Transport Infrastructures.

Jean-Pierre MAGNAN, Jean-Paul DURANTHON,

Patrick JOFFRIN, François DEPARDON,

Dominique Allagnat, François Lemaître,

Philippe Évenat, Philippe Le STER

1.1. Introduction. . . . . . . . . . . . . . . . . 3

1.2. Development of transportable ultra wide-band radar . . . . . . . 6

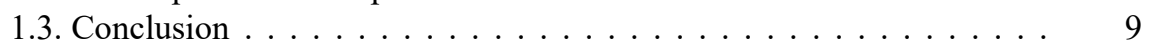

1.4. Acknowledgments. . . . . . . . . . . . . . . 9

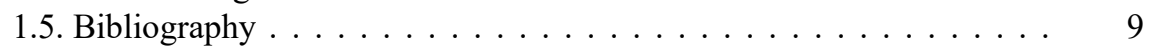

Chapter 2. Intelligent Compaction Technology

for Geomaterials: A Demonstration Project . . . . . . . . . . . . . 11

António GOMEs CORREIA, Manuel PARENTE

2.1. Introduction. . . . . . . . . . . . . . . . . . . . . 11

2.2. Demonstration project $\ldots \ldots \ldots \ldots \ldots \ldots \ldots \ldots \ldots$

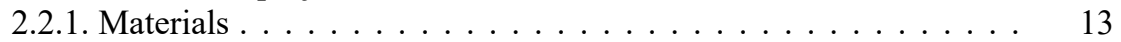

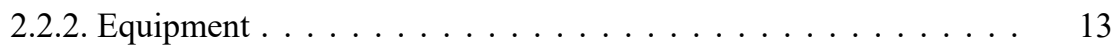


2.2.3. Foundation evaluation and layer construction . . . . . . . . . . 14

2.2.4. Results and discussion . . . . . . . . . . . . . . . . . . . . . . . 16

2.2.5. Soil-rockfill layers . . . . . . . . . . . . . . . . . . . . . . . . . . . .

2.2.6. Soil layer. . . . . . . . . . . . . . . . . . . . . . . . . . . . . . . . . 21

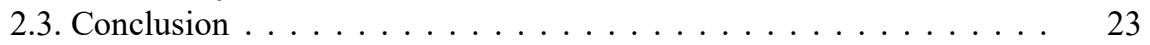

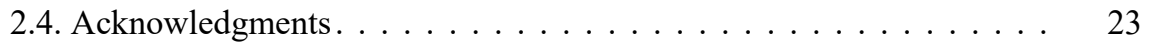

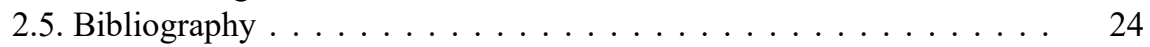

\section{Chapter 3. Geotechnical Challenges Related} to Transport Infrastructures on Sensitive

Soft Clay Deposits . . . . . . . . . . . . . . . . . . . . . . 27

Vikas THAKUR, Bjorn Kristoffer DOLVA

3.1. Nomenclature . . . . . . . . . . . . . . . . . . . . . . . . 27

3.2. Introduction. . . . . . . . . . . . . . . . . . 28

3.3. Challenges related to the characterization of sensitive clays . . . . . 29

3.3.1. Sample disturbance. . . . . . . . . . . . . . . . . . . 31

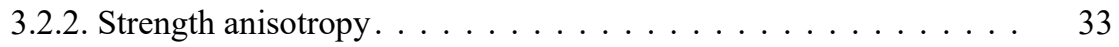

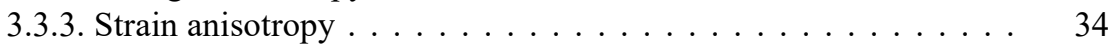

3.4. Challenges related to the assessment of safety margins . . . . . . 35

3.5. Post-failure assessment . . . . . . . . . . . . . . . . 37

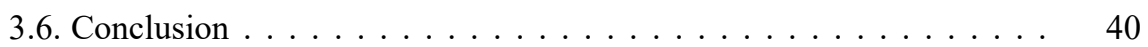

3.7. Acknowledgments. . . . . . . . . . . . . . . 40

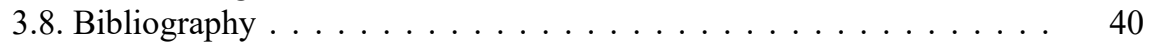

\section{Chapter 4. Performance Control of}

Bituminous Mixtures with a High RAP Content . . . . . . . . 43

Frédéric Delfosse, Ivan DrouADAINE,

Stéphane FAUCON DUMONT and Sabine LARGEAUD

4.1. Introduction. . . . . . . . . . . . . . . . . . 43

4.2. Impact of the high RAP content in the

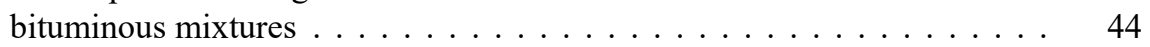

4.3. Normative context. . . . . . . . . . . . . . . 45

4.4. Development of a system to measure the

blending degree of the RAP . . . . . . . . . . . . . . . 47

4.5. Impact of the RAP content on the mechanical properties of the recovered binder . . . . . . . . . . . . . . 49

4.6. Correlation between laboratory and jobsite production . . . . . . . 52

4.7. Rheological model. . . . . . . . . . . . . . . . . . 53

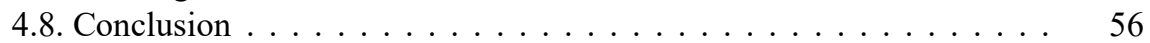

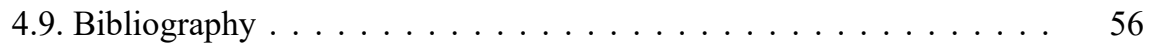


Chapter 5. Integration of Materials Sciencebased Performance Models into PMS

Altred WeNINGER-VYCUDIL, Michael Wistuba, Goran MLADENOVIC, Johan LitZKA, Axel WALTHER and Alexander ALISOV

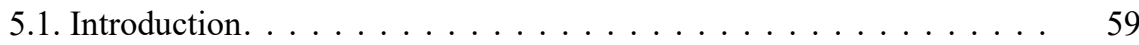

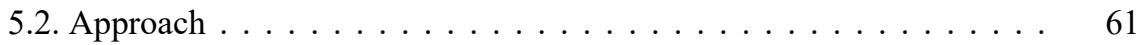

5.3. Integration of material-based performance

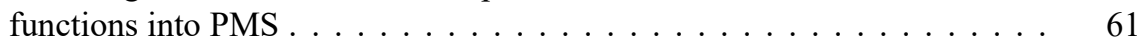

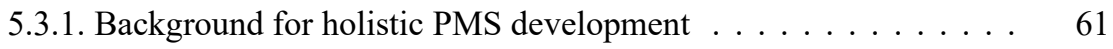

5.3.2. Laboratory testing and structural modeling I. . . . . . . . . . 63

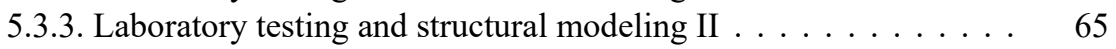

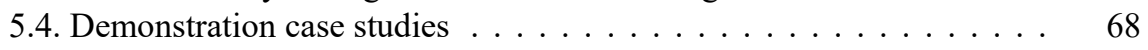

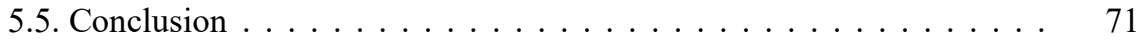

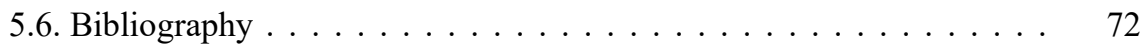

\section{Chapter 6. Decision Aid Model for}

Asphalt Mixture Choice . . . . . . . . . . . . . . . . . . 75

Nicolas BUECHE and André-Gilles DUMONT

6.1. Background and objectives $\ldots \ldots \ldots \ldots \ldots \ldots \ldots \ldots \ldots$

6.2. Model architecture. . . . . . . . . . . . . . . . . . 76

6.2.1. Lifecycle inventory and performance indicators (model part 1$) \ldots \ldots \ldots \ldots \ldots \ldots \ldots \ldots$

6.2.2. Global evaluation (model part 2). . . . . . . . . . . . . . . 79

6.3. Model implementation and results . . . . . . . . . . . 81

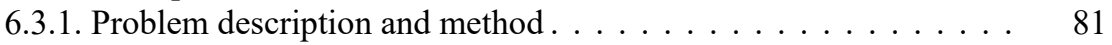

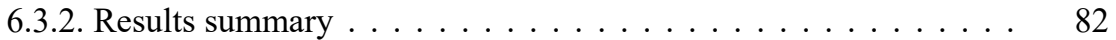

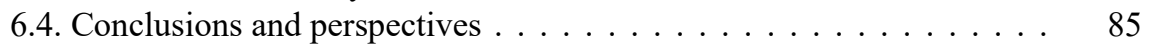

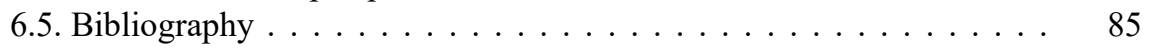

Chapter 7. Experimental Study of Binder-Filler Interaction Using the Modified Multiple

Stress-Strain Creep Recovery Test . . . . . . . . . . . . . . 87

Mahmoud ELNASRI, Nick THOM and Gordon AIREY

7.1. Introduction. . . . . . . . . . . . . . . . . . . . . 87

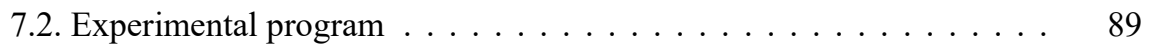

7.2.1. Materials . . . . . . . . . . . . . . . . . . 89

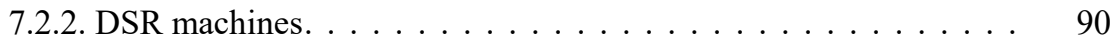

7.2.3. Sample preparation. . . . . . . . . . . . . . . . 91

7.3. The development of multiple stress-strain creep recovery test $\ldots \ldots \ldots \ldots \ldots \ldots \ldots \ldots \ldots \ldots$ 


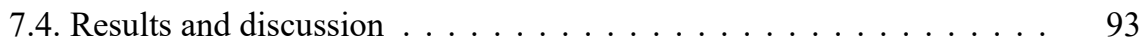

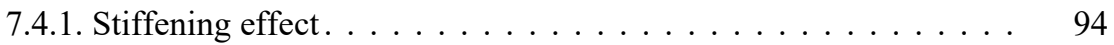

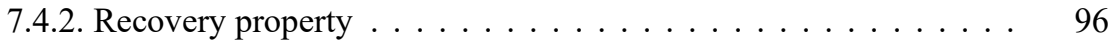

7.5. Discussion and conclusions . . . . . . . . . . . . . . 98

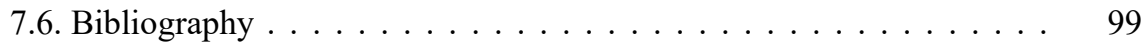

\section{Chapter 8. Reliability of New Shear Design}

Equations for FRP-strengthened Concrete

Bridge Girders

Ayman M. OKeIL, Abdeldjelil BelarBI and Daniel A. KUCHMA

8.1. Nomenclature . . . . . . . . . . . . . . . . . . . . . . . 101

8.2. Introduction. . . . . . . . . . . . . . . . . . . . 102

8.3. Shear strengthening using composites . . . . . . . . . . . . . 104

8.3.1. Design of FRP-strengthened concrete beams . . . . . . . . . 104

8.3.2. Effective FRP strain, $\varepsilon_{f e} \ldots \ldots \ldots \ldots \ldots \ldots \ldots \ldots$

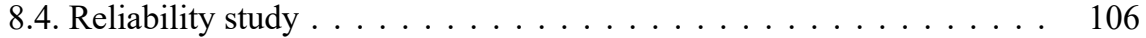

8.4.1. Design space. . . . . . . . . . . . . . . . . . . . . 107

8.4.2. Design parameters as random variables. . . . . . . . . . . 108

8.4.3. Reliability formulation and analysis . . . . . . . . . . . . 109

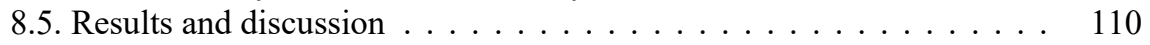

8.6. Conclusions. . . . . . . . . . . . . . . . . . . 112

8.7. Acknowledgments. . . . . . . . . . . . . . . . . . 112

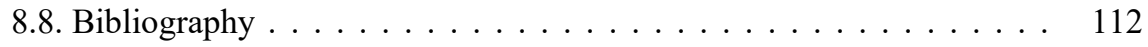

\section{Chapter 9. Experimental Investigation and}

Modeling of the Bond between Aramid

Fiber-reinforced Polymer Bars and Concrete . . . . . . . . . . . . 115

Arnaud Rolland, Sylvain ChataIGNER, Karim BenZARTI,

Marc QUIERTANT, Pierre ARGOUL and Jean-Marc PAUL

9.1. Introduction. . . . . . . . . . . . . . . . . . . . . 115

9.2. Material properties at ambient temperature . . . . . . . . . 116

9.2.1. Microscopic observations . . . . . . . . . . . . . . . . 116

9.2.2. Tensile tests . . . . . . . . . . . . . . . . . . . 117

9.2.3. Glass transition temperature $\ldots \ldots \ldots \ldots \ldots \ldots \ldots$

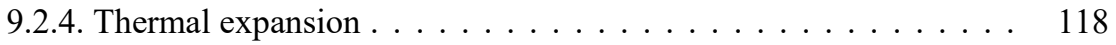

9.3. Bond tests . . . . . . . . . . . . . . . . . . . . 119

9.3.1. Geometry and preparation of the pull-out specimens . . . . . . 120

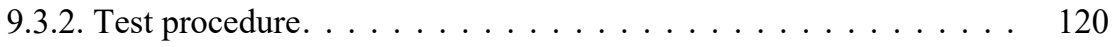

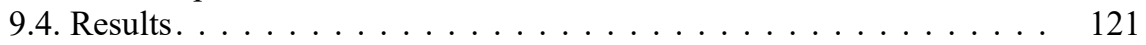

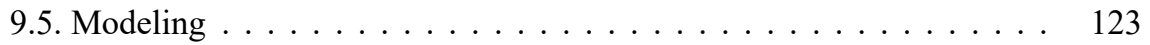

9.5.1. Analytical law . . . . . . . . . . . . . . . . . 123 
9.5.2. Parameter identification method . . . . . . . . . . . . . . . 124

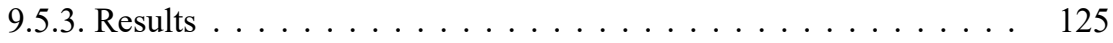

9.6. Conclusions. . . . . . . . . . . . . . . . . . . . . 126

9.7. Acknowledgments . . . . . . . . . . . . . . . . . . . . . . . . . . . . . . . . . . . . . 127

9.8. Bibliography . . . . . . . . . . . . . . . . . . . . 127

Chapter 10. Innovative Use of FRP for

Sustainable Precast Concrete Structures . . . . . . . . . . . . . . 129

Sami RIZKALLA

10.1. Introduction . . . . . . . . . . . . . . . . . . . . . . . . . . 129

10.2. Double-tees . . . . . . . . . . . . . . . . 130

10.3. Precast concrete wall panels . . . . . . . . . . . . . . 133

10.3.1. Fully composite sandwich wall panels. . . . . . . . . . . 133

10.3.2. Non-composite sandwich wall panels . . . . . . . . . . . 134

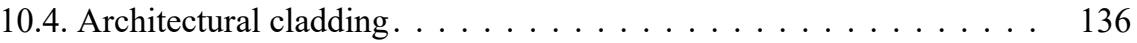

10.5. Precast concrete piles (CFFT) _ . . . . . . . . . . . . . . 136

10.6. Bridge girders. . . . . . . . . . . . . . . . . . . 139

10.7. Future opportunities . . . . . . . . . . . . . . . . . . 140

10.8. Conclusions . . . . . . . . . . . . . . . . . . . . . 141

10.9. Acknowledgments . . . . . . . . . . . . . . . . . . . . 142

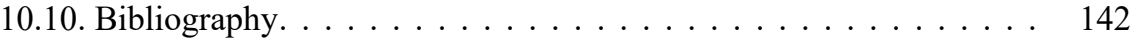

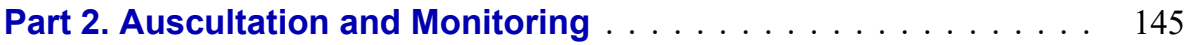

Chapter 11. 3D Extraction of the Relief

of Road Surface through Image Analysis. . . . . . . . . . . . . . 147

Majdi KHOUDEIR and Benjamin BRINGIER

11.1. Introduction . . . . . . . . . . . . . . . . . . . . . . . . 147

11.2. Photometric models . . . . . . . . . . . . . . . . . . . . . . 148

11.2.1. Lambert's model . . . . . . . . . . . . . . . . . . . . 148

11.2.2. Phong model . . . . . . . . . . . . . . . . . . . . . 149

11.2.3. Blinn-Phong model. . . . . . . . . . . . . . . . 150

11.2.4. Mixed model. . . . . . . . . . . . . . . . . . 150

11.3. Extraction of gradient field . . . . . . . . . . . . . . . 151

11.3.1. The suggested global approach . . . . . . . . . . . . . 151

11.3.2. Gradient extraction based on the Lambertian model . . . . . . . 151

11.3.3. Gradient field of specular area . . . . . . . . . . . . . 153

11.4. Relief extraction . . . . . . . . . . . . . . . . . 154

11.4.1. Limits of the classical approach . . . . . . . . . . . . 154

11.4.2. Results of the suggested approach . . . . . . . . . . 155

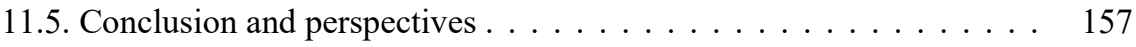

11.6. Bibliography . . . . . . . . . . . . . . . . . . . 157 
Chapter 12. Measurement Error Models (MEMs)

Regression Method to Harmonize Friction Values

from Different Skid Testing Devices

Azzurra Evangelisti, Samer W. Katicha,

Edgar DE LEÓN IZEPPI, Gerardo W. FLINTSCH,

Mauro D'APUZZO and Vittorio NiCOLOSI

12.1. Introduction . . . . . . . . . . . . . . . . . . . . . . . . . . . . 159

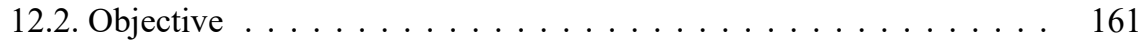

12.3. MEM: background and modeling . . . . . . . . . . . . . . . . 161

12.3.1. MEM modeling for pavement friction applications . . . . . . . . . 162

12.4. Data collection . . . . . . . . . . . . . . . . . . . . . . . . . . . . . . . . . . 165

12.5. Results and analysis . . . . . . . . . . . . . . . . . 166

12.5.1. Repeatability evaluation . . . . . . . . . . . . . . . . 166

12.5.2. Relationship between two of the same

measuring principles devices . . . . . . . . . . . . . . . 166

12.5.3. Relationship between two different

measuring principles devices . . . . . . . . . . . . . . . . . . . . . 168

12.6. Conclusion . . . . . . . . . . . . . . . . . . . . . . . . . 171

12.7. Acknowledgments . . . . . . . . . . . . . . . . . . . . . . . . . . . . . . . . . . . . 172

12.8. Bibliography . . . . . . . . . . . . . . . . . . 172

Chapter 13. Accurate and Up-to-Date Evaluation

of Extreme Load Effects for Bridge Assessment . . . . . . . . . . 175

Xiaoyi ZHOU, Franziska SCHMIDT,

François TOUTLEMONDE and Bernard JACOB

13.1. Introduction . . . . . . . . . . . . . . . . . . . . . . . . . . . . . . . . . 175

13.2. WIM data files . . . . . . . . . . . . . . . . . . . . . . . 178

13.3. Extrapolated values for the considered WIM data . . . . . . . . . . . . . 181

13.4. Conclusion . . . . . . . . . . . . . . . . . . . . . . . . . . . . . . . . . . . . 182

13.5. Acknowledgments . . . . . . . . . . . . . . . . . . . . . . . . . . . . . . . . . . . . 183

13.6. Bibliography . . . . . . . . . . . . . . . . . 183

Chapter 14. Transportation Infrastructure

Monitoring Using Satellite Remote Sensing . . . . . . . . . . . . 185

Edward HOPPE, Brian BRUCKNO, Elizabeth CAMPBELL,

Scott ACtOn, Andrea VACCARI, Michael StUECHELI,

Adrian BOHANE, Giacomo FALORNI and Jessica MORGAN

14.1. Introduction . . . . . . . . . . . . . . . . . . . . . . . 185

14.2. Purpose and scope . . . . . . . . . . . . . . . . . . . . . . . . . . . . . . . . . . 187

14.3. Methodology . . . . . . . . . . . . . . . . . . . . . 187

14.3.1. Selection of processing algorithm . . . . . . . . . . . 187 
14.3.2. Selection of satellite system . . . . . . . . . . . . . . . . . . . . . . . 187

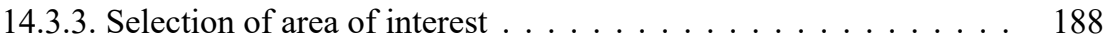

14.4. Results . . . . . . . . . . . . . . . . . . . . . . . . . 188

14.4.1. Data . . . . . . . . . . . . . . . . . . . . . . . . . . . . . . . . . . 188

14.4.2. Scatterer density and distribution. . . . . . . . . . . . . . . . . . . . . . . . 189

14.4.3. Sinkhole detection. . . . . . . . . . . . . . . . . . . . 190

14.4.4. Rock slope monitoring . . . . . . . . . . . . . . . . . . . . . . . . . . . . . . 193

14.4.5. Bridge monitoring. . . . . . . . . . . . . . . . . . . . . . . . . . . . . . . . . . . . . . . . 194

14.4.6. Pavement monitoring . . . . . . . . . . . . . . . . . . . . . . . . . . . . . . . . . . . . 194

14.7. Discussion. . . . . . . . . . . . . . . . . . . . 196

14.8. Conclusions . . . . . . . . . . . . . . . . . . . . . . . . . . . . . . . . . . . . 197

14.9. Acknowledgments . . . . . . . . . . . . . . . . . . . . . . . . . . . . . . . . . . . . . . . . . . . . . . .

14.10. Disclaimer . . . . . . . . . . . . . . . . . . . . 198

14.11. Bibliography. . . . . . . . . . . . . . . . . . . . . 198

Chapter 15. Monitoring of Scour Critical

Bridges using Changes in the Natural

Frequency of Vibration of Foundation Piles:

A Preliminary Investigation . . . . . . . . . . . . . . . . . . 199

Luke J. PRENDERGAST and Kenneth GAVIN

15.1. Nomenclature. . . . . . . . . . . . . . . . . . . . . . . 199

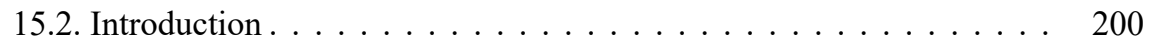

15.3. Scour monitoring using fixed instruments . . . . . . . . . . 201

15.4. Scour monitoring using structural response measurement. . . . . . 203

15.5. Field investigation at UCD dense sand test site . . . . . . . . . 204

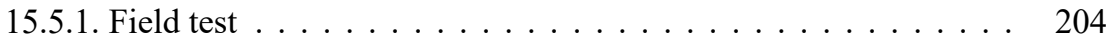

15.5.2. Numerical modeling . . . . . . . . . . . . . . 205

15.6. Results and conclusions. . . . . . . . . . . . . . . 207

15.7. Acknowledgments . . . . . . . . . . . . . . . . . 208

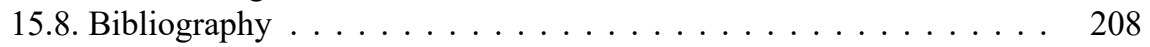

\section{Chapter 16. Evaluation of Multilayer}

Pavement Viscoelastic Properties from

Falling Weight Deflectometer using Neural Networks . . . . . . . . 211

José Manuel GONZALEZ, Josep Maria CARBONELL and

Wouter VAN BIJSTERVELD

16.1. Nomenclature. . . . . . . . . . . . . . . . . . . . . . . 211

16.2. Introduction . . . . . . . . . . . . . . . . . . . 212

16.3. Methodology . . . . . . . . . . . . . . . . . . . 213

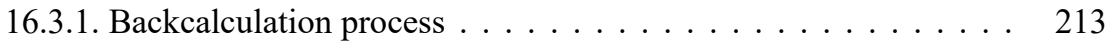

16.3.2. Constitutive model . . . . . . . . . . . . . . . . 214

16.3.3. Development of an artificial neural network . . . . . . . 215 
16.4. Calibration process. . . . . . . . . . . . . . . 216

16.4.1. Input data: load function and deflection curves . . . . . . . . . . . . 216

16.4.2. Numerical simulation of the FWD problem . . . . . . . . . . . 218

16.4.3. Calibration process results . . . . . . . . . . . . 220

16.5. Backcalculation process: artificial neural network . . . . . . . . 222

16.6. Conclusions . . . . . . . . . . . . . . . . . . 223

16.7. Acknowledgments . . . . . . . . . . . . . . . . . . . . . . . . . . . . . . . 224

16.8. Bibliography . . . . . . . . . . . . . . . . . 224

Chapter 17. Accuracy of Ground-penetrating Radar in Pavement Thickness Evaluation: Impact of Interpretation Errors

Anne LalagüE, Matthew A. LEBENS and Inge HoFF

17.1. Introduction . . . . . . . . . . . . . . . . . . . . . . 227

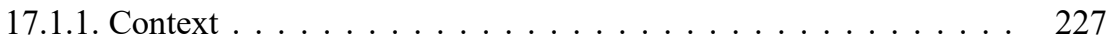

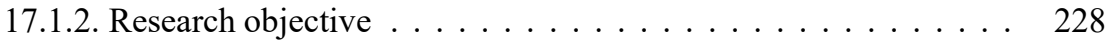

17.2. Ground-penetrating radar technology. . . . . . . . . . . . 228

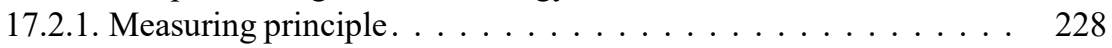

17.2.2. GPR systems . . . . . . . . . . . . . . . . . . 229

17.3. Data collection and interpretation . . . . . . . . . . . 230

17.3.1. GPR measurements . . . . . . . . . . . . . . . . 230

17.3.2. Soil sample collection . . . . . . . . . . . . . . 231

17.3.3. Calibration . . . . . . . . . . . . . . . . . . 231

17.3.4. Data interpretation . . . . . . . . . . . . . 232

17.4. Results . . . . . . . . . . . . . . . . . . . 233

17.4.1. Hot mix asphalt layer. . . . . . . . . . . . . . . . . 233

17.4.2. Base layer. . . . . . . . . . . . . . . . . . 235

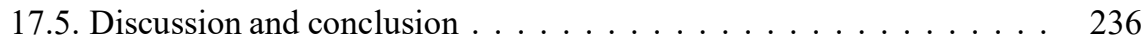

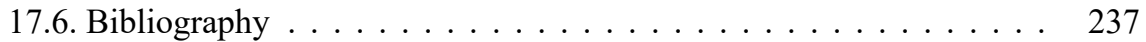

Chapter 18. Full-scale Test on Prefabricated

Slabs for Electrical Supply by Induction of Urban Transport Systems

Mai-Lan NGUYEN, Pierre HORNYCH, Jean-Pierre KeRZRÉHO and Sergio PEREZ

18.1. Introduction . . . . . . . . . . . . . . . . . . 239

18.2. Design of the full-scale test. . . . . . . . . . . . . . . 241

18.3. Construction of the full-scale test and solution

for installation of the prefabricated slabs . . . . . . . . . . . . 243

18.3.1. Procedure for installation of the slabs A, B, C and D . . . . . 243

18.3.2. Procedure for installation of slab E. . . . . . . . . . . 245 
18.4. Test conditions and parameters measured during the test . . . . . . . . 245

18.4.1. Test conditions. . . . . . . . . . . . . . . . . 245

18.4.2. Parameters measured during the test . . . . . . . . . . . . . . . . 246

18.5. First results and interpretation . . . . . . . . . . . . . . 247

18.5.1. Deflections of concrete slab pavement

measured at joints . . . . . . . . . . . . . . . . . 247

18.5.2. Vertical subgrade strains under the

prefabricated slabs . . . . . . . . . . . . . . . . . . 249

18.5.3. Horizontal strains at the bottom of

the prefabricated slab . . . . . . . . . . . . . . . . . . 250

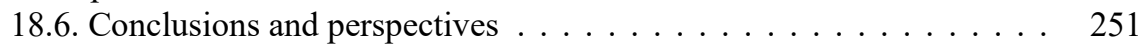

18.7. Bibliography . . . . . . . . . . . . . . . . . 252

Part 3. Durability and Maintenance Repair . . . . . . . . . . . . . . . 253

Chapter 19. The Poroelastic Road

Surface (PERS): Is the $10 \mathrm{~dB}$ Reducing

Pavement within Reach?

Luc Goubert, Hans BendTsen, Anneleen Bergiers,

Björn KALMAN and Darko KOKOT

19.1. Introduction . . . . . . . . . . . . . . . . . . . . . . . . 255

19.2. The PERSUADE project . . . . . . . . . . . . . . . . . . . . . 257

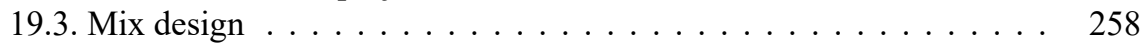

19.4. Safety issues . . . . . . . . . . . . . . . . . . . . . . . . 260

19.5. Cost-benefit analysis . . . . . . . . . . . . . . . . . . . . . . . . . . 261

19.6. Test tracks on the road . . . . . . . . . . . . . . . . . 262

19.6.1. Test tracks in Denmark. . . . . . . . . . . . . . . . . . . . . . . . . . 262

19.6.2. Test tracks in Belgium . . . . . . . . . . . . . . . . . . . . . 265

19.6.3. Test tracks in Slovenia . . . . . . . . . . . . . . . . . . . . . . 266

19.7. Conclusions . . . . . . . . . . . . . . . . . . . . 267

19.8. Acknowledgments . . . . . . . . . . . . . . . . . . . . 267

19.9. Bibliography . . . . . . . . . . . . . . . . . 267

Chapter 20. Modeling Subjective Condition

Data of Asphalt Surfaced Urban Pavements . . . . . . . . . . . 269

Rayya HASSAN, Oliver LIN and Amutha THANANJEYAN

20.1. Introduction . . . . . . . . . . . . . . . . . . . . . . . . . . . . . . . . 269

20.2. Regression and Markov chain modeling . . . . . . . . . . . . . . . . . 270

20.3. Data description and preparation . . . . . . . . . . . . 271

20.3.1. Calculation of SIR . . . . . . . . . . . . . . . . 272

20.4. Modeling using deterministic regression analysis . . . . . . . . . 273 
20.5. Modeling surfacing deterioration using Markov chains . . . . . . . . . . . . 274

20.5.1. Number of states . . . . . . . . . . . . . . . . . . 274

20.5.2. State vector. . . . . . . . . . . . . . . . . . . . 275

20.5.3. Stage or duty cycle definition . . . . . . . . . . . . . . . . 276

20.5.4. The transition probability matrix . . . . . . . . . . . . . 276

20.6. Markov models for sample AC network . . . . . . . . . . . . . . . 279

20.6.1. Validation of Markov models . . . . . . . . . . . . . . . . 281

20.6.2. Markov models using initial vector and average condition values . . . . . . . . . . . . . . . . 281

20.7. Comparison of models . . . . . . . . . . . . . . . 283

20.8. Conclusions . . . . . . . . . . . . . . . . . . . . . . . . . . . . . . . . . . . . . 284

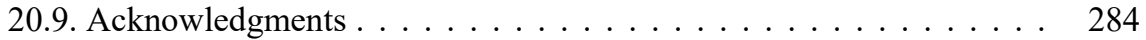

20.10. Bibliography. . . . . . . . . . . . . . . . . . . 284

\section{Chapter 21. Modeling of Aging of}

Low-noise Road Surfaces . . . . . . . . . . . . . . . . . . . . . 287

Gijsjan VAN BLOKLAND, Ronald VAN LOON

and Christiaan TOLLENAAR

21.1. Introduction . . . . . . . . . . . . . . . . . . . . . . 287

21.2. Noise reduction over time . . . . . . . . . . . . . . . . 289

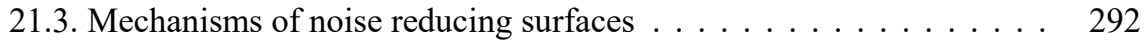

21.4. Deterioration process . . . . . . . . . . . . . . . . . 294

21.5. Parameters. . . . . . . . . . . . . . . . . . . . . . 295

21.6. Discussion. . . . . . . . . . . . . . . . . . . . . . . . 297

21.7. Acknowledgments . . . . . . . . . . . . . . . . . . . . . 299

21.8. Bibliography . . . . . . . . . . . . . . . . . . . . . 299

\section{Chapter 22. Evaluation of Load-carrying}

Capacity of Asphalt Superstructures from

Deflection Measurements . . . . . . . . . . . . . . . . . . . . . 301

J. Stefan BALD and Anh-Duc NGUYEN

22.1. Nomenclature. . . . . . . . . . . . . . . . . . . . . . . . 301

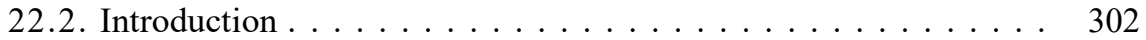

22.3. Theoretical analysis. . . . . . . . . . . . . . 303

22.3.1. Implementation of plate theory for

determining deflection at surface of an asphalt structure . . . . . 303

22.3.2. Interpretation the load behavior of asphalt

pavement slab as load distributing layer of superstructure . . . . . 306

22.3.3. Back-calculation for evaluating FWD

data of asphalt structure using regression method . . . . . . . . 309 
22.4. Validation of theoretical analysis . . . . . . . . . . . . . . 310

22.4.1. Experimental data . . . . . . . . . . . . . . . . 310

22.4.2. Verification of the regressive back-calculated

mechanical parameters ... . . . . . . . . . . . . . 312

22.5. Conclusion and recommendation . . . . . . . . . . . . . . 314

22.6. Bibliography . . . . . . . . . . . . . . . . 315

Chapter 23. Durable Pothole Repairs . . . . . . . . . . . . . . . . . . . . . 317

Cliff NiCHOLLS, Kathrin KUBANEK,

Carsten Karcher, Andreas HARTMANN,

Adewole ADESIYUn, Aleksander IPAVEC,

Jozef KOMAC̆KA and Erik NIELSEN

23.1. Introduction . . . . . . . . . . . . . . . . . . . 317

23.2. Definition of the term "pothole" . . . . . . . . . . . . . . . 318

23.3. Tests and evaluation methods for use in

the laboratory and in situ. . . . . . . . . . . . . . . . . . . . . . . . . . . . . . . 319

23.4. Existing standards, techniques, materials and

experience with them on the European market. . . . . . . . . . . . . 320

23.5. Experience from trial sections . . . . . . . . . . . . . . . . 322

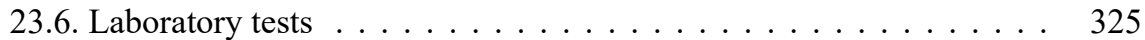

23.6.1. Materials tested . . . . . . . . . . . . . . . . . . . . . . . 325

23.6.2. Cold asphalts . . . . . . . . . . . . . . . . . . . . . . . . . . . . . . 325

23.6.3. Analysis of results. . . . . . . . . . . . . . . . . . . . 327

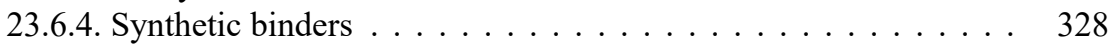

23.7. Whole lifecycle costs and benefits . . . . . . . . . . . . . . . 329

23.8. Conclusions . . . . . . . . . . . . . . . . . . . . . 331

23.9. Acknowledgments . . . . . . . . . . . . . . . . . . . . . . . . . . . . . 332

23.10. Bibliography. . . . . . . . . . . . . . . . 332

Chapter 24. Application of Multicriteria Assessment

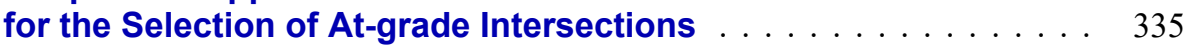

Jan HRADIL, Michal UHLÍK and Petr SLABÝ

24.1. Introduction . . . . . . . . . . . . . . . . . . . . 335

24.2. MCA application in the choice of

at-grade intersections . . . . . . . . . . . . . . . . . . . . . . 336

24.2.1. MCA fundamentals . . . . . . . . . . . . . . . . . . . . . . . . . . . . . . . . . 336

24.2.2. MCA alternatives . . . . . . . . . . . . . . . . . . . . . . . . . . . . . . . . . 337

24.2.3. Decision tree . . . . . . . . . . . . . . . . . . . . . . . . . . . . . . . . 338

24.2.4. Evaluated criteria in MCA . . . . . . . . . . . . . . . . . . . . . . . . . . . . . . . . . . . . . .

24.2.5. Weight of criteria . . . . . . . . . . . . . . . 341 
24.3. Selected intersection and its alternative design . . . . . . . . . . . . . . . . . . 342

24.3.1. Existing state. . . . . . . . . . . . . . . . . . 342

24.3.2. Design of layout alternatives and their MCA evaluation . . . . . . 344

24.3.3. Evaluation of the MCA analysis results . . . . . . . . . . . . . . . . . . 346

24.4. Conclusion . . . . . . . . . . . . . . . . . . . . 346

24.5. Acknowledgments . . . . . . . . . . . . . . . . . . . 347

24.6. Bibliography . . . . . . . . . . . . . . . . . . 347

Chapter 25. Low-energy and Environmentally-friendly

Solutions for Road Maintenance. . . . . . . . . . . . . . . . . . . . . 349

Bernard ECKMANN, Frédéric DELFOSSE,

Philippe POILANE and Bruno TAILLIS

25.1. Introduction .

25.2. Flexible bituminous mixtures for the

maintenance of flexible low traffic roads . . . . . . . . . . . . . . . 350

25.2.1. The specific needs of maintenance on low traffic roads . . . . . 350

25.2.2. Wearing course mixes . . . . . . . . . . . . . . . . . . 351

25.2.3. Re-profiling works . . . . . . . . . . . . . . . . . . . . . . . . . . 353

25.2.4. Small repair works . . . . . . . . . . . . . . . . . 353

25.3. Emulsion-based cold bituminous mixtures. . . . . . . . . . . . . . 354

25.3.1. Specific problems raised by emulsion-based mixes to be used in wearing courses . . . . . . . . . . . . . . . . . 354

25.3.2. Development of emulsion-based wearing course mixes . . . . . 355

25.3.3. Experimental trial sections. . . . . . . . . . . . . . . . . . . 356

25.3.4. Experimental trials: what they told us . . . . . . . . . . . . . 360

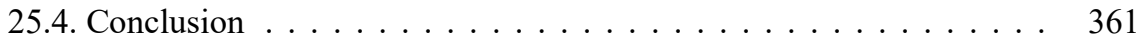

25.5. Bibliography . . . . . . . . . . . . . . . . 362

Chapter 26. 3D Longitudinal and Transverse

Cracking and the Influence of Non-Uniform

Contact Pressure on the Stress Intensity

Factors of these Cracks.

Dermot B. CASEY, James R. GRENFELL and Gordon AIREY

26.1. Introduction . . . . . . . . . . . . . . . . . . . . . 365

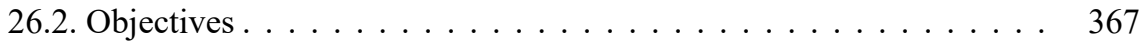

26.3. Methodology . . . . . . . . . . . . . . . . . . . . 367

26.3.1. Mesh setup . . . . . . . . . . . . . . . . . . . . 367

26.3.2. Loading scenarios . . . . . . . . . . . . . . . . . . . . 369

26.3.3. Stress intensity factor calculations . . . . . . . . . . . . 370

26.4. Results and discussion. . . . . . . . . . . . . . . . . 371

26.4.1. Longitudinal crack . . . . . . . . . . . . . . . . 371

26.4.2. Transverse crack. . . . . . . . . . . . . . . . . . 373 
26.5. Conclusions . . . . . . . . . . . . . . . . . . . . 376

26.6. Acknowledgments . . . . . . . . . . . . . . . . . . . 377

26.7. Bibliography . . . . . . . . . . . . . . . . . . . 377

Chapter 27. Selecting a Road Network Maintenance

Strategy to Achieve the Operator's Objectives . . . . . . . . . . . 381

Pierre HANKACH and Philippe LEPERT

27.1. Introduction . . . . . . . . . . . . . . . . . . . 381

27.2. Maintenance strategies . . . . . . . . . . . . . . 382

27.2.1. Definition. . . . . . . . . . . . . . . . . . 382

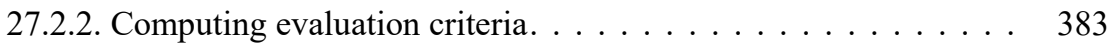

27.2.3. Evolution models of distress indicators . . . . . . . . . . . 384

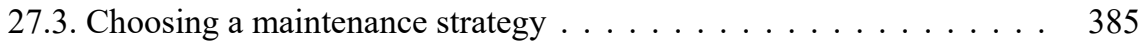

27.4. An ELECTRE III-based approach. . . . . . . . . . . . . . . . 386

27.4.1. Introduction. . . . . . . . . . . . . . . . . . . 386

27.4.2. Pseudo-criteria . . . . . . . . . . . . . . . . . . . . . 387

27.4.3. Concordance and discordance indices . . . . . . . . . . 388

27.4.4. Degree of credibility . . . . . . . . . . . . . . . . . . 389

27.4.5. Distillation procedures . . . . . . . . . . . . . . . . . 390

27.5. Example . . . . . . . . . . . . . . . . . . . . 391

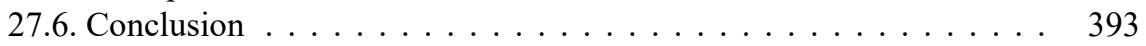

27.7. Bibliography . . . . . . . . . . . . . . . . . . . 394

List of Authors . . . . . . . . . . . . . . . . . . . . . . . . 397

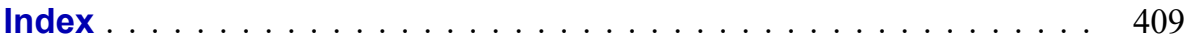

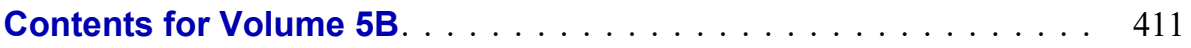




\section{Preface}

The transport sector is very much concerned about environmental adaptation and mitigation issues. Most of these are related to the objective of curbing GHG emission by $20 \%$ by 2020 , alternative energy and energy savings, sustainable mobility and infrastructures, safety and security, etc. These objectives require the implementation of advanced research works, to develop new policies, and to adjust education and industrial innovations.

The theme and slogan of the Transport Research Arena held in Paris (TRA2014) were respectively: "Transport Solutions: From Research to Deployment" and "Innovate Mobility, Mobilise Innovation". Top researchers and engineers, as well as private and public policy and decision-makers, were mobilized to identify and take the relevant steps to implement innovative solutions in transport. All surface modes were included, including walking and cycling, as well as cross modal aspects.

Policies, technologies and behaviors must be continually adapted to new constraints, such as climate change, the diminishing supply of fossil fuels, the economic crisis, the increased demand for mobility, safety and security, i.e. all the societal issues of the $21^{\text {st }}$ Century. Transport infrastructures and materials, modal share, co-modality, urban planning, public transportation and mobility, safety and security, freight, logistics, ITS, energy and environment issues are the subject of extensive studies, research works and industrial innovations that are reported in this series of books.

This book is part of a set of six books called the Research for Innovative Transports set. This collection presents an update of the latest academic and applied research, case studies, best practices and user perspectives on transport carried out in Europe and worldwide. The presentations made during TRA2014 reflect on them. The TRAs are supported by the European Commission (DG-MOVE and DG-RTD), the Conference of European Road Directors (CEDR), and the modal European 
platforms, ERRAC (rail), ERTRAC (road), WATERBORNE, and ALICE (freight), and also by the European Construction Technology Platform (ECTP) and the European Transport Research Alliance (ETRA).

The volumes are made up of a selection of the best papers presented at TRA2014. All papers were peer reviewed before being accepted at the conference, and were then selected by the editors for the purpose of the present collection. Each volume contains complementary academic and applied inputs provided by highly qualified researchers, experts and professionals from all around the world.

Each volume of the series covers a strategic theme of TRA2014.

Volume 1, Energy and Environment, presents recent research works around the triptych "transports, energy and environment" that demonstrate that vehicle technologies and fuels can still improve, but it is necessary to prepare their implementation (electro-mobility), think about new services and involve enterprises. Mitigation strategies and policies are examined under different prospective scenarios, to develop and promote alternative fuels and technologies, multi-modality and services, and optimized transport chains whilst preserving climate and the environment. Evaluation and certification methodologies are key elements for assessing air pollution, noise and vibration from road, rail and maritime transports and their impacts on the environment. Different depollution technologies and mitigation strategies are also presented.

Volume 2, Towards Innovative Freight and Logistics, analyzes how to optimize freight movements and logistics, introduces new vehicle concepts, points out the governance and organization issues, and proposes an assessment framework.

Volumes 3 and 4 are complementary books covering the topic of traffic management and safety.

Volume 3, Traffic Management, starts with a survey of data collection processes and policies and then shows how traffic modeling and simulation may resolve major problems. Traffic management, monitoring and routing tools and experience are reported and the role of traffic information is highlighted. Impact assessments are presented.

Volume 4, Traffic Safety, describes the main road safety policies, accident analysis and modeling. Special focus is placed on the safety of vulnerable road users. The roles of infrastructure and ITS on safety are analyzed. Finally railway safety is focused upon. 
Volume 5, Materials and Infrastructures, split into two sub-volumes, investigating geotechnical issues, and pavement materials' characterization, innovative materials, technologies and processes, and introducing new techniques and approaches for auscultation and monitoring. Solutions to increase the durability of infrastructures and to improve maintenance and repair are shown, for recycling as well as for ensuring the sustainability of the infrastructures. Specific railways and inland navigation issues are addressed. A focus is put on climate resilient roads.

Volume 6, Urban Mobility and Public Transport, highlights possible innovations in order to improve transports and the quality of life in urban areas. Buses and twowheelers could be a viable alternative in cities if they are safe and reliable. New methodologies are needed to assess urban mobility through new survey protocols, a better knowledge of user behavior or taking into account the value of travel for public transport. The interactions between urban transport and land planning are a key issue. However, these interactions have to be better assessed in order to propose scenarios for new policies.

Bernard JACOB, Chair of the TRA2014 Programme Committee Jean-Bernard KOVARIK, Chair of the TRA2014 Management Committee 


\section{Acknowledgments}

The European Commission, DG MOVE and RTD, the Conference of European Road Directors (CEDR), the European Road Transport Research Advisory Council (ERTRAC), the European Rail Research Advisory Council (ERRAC) and the European technology platform WATERBORNE-TP are acknowledged for their support and active contribution to the Programme Committee of the TRA2014, in charge of reviewing and selecting the papers presented at the conference, which forms the main input of this volume.

The French Institute of Science and Technology for Transport, Development and Networks (IFSTTAR) is acknowledged for having organized the TRA2014, in which 600 high-quality papers were presented, successfully.

Anne Beeldens, Pierre Marchal, Manuel Pereira, and Jon Krokeborg; coordinators of the topic on Materials and Infrastructure; all the other members of the Programme Committee; the reviewers who actively contributed to review and select the papers; and the authors who wrote them are acknowledged for their great job that produced the material for this volume.

Joëlle Labarrère, secretary of the Programme Committee of TRA2014, is acknowledged for her valuable help to the editors and for her support to prepare this volume.

\section{Francesca La Torre}

Professor Francesca La Torre is a Full Professor of roads, railways and airports at the University of Florence (Italy). She has been working in the field of transportation infrastructures for over 20 years. She obtained her $\mathrm{PhD}$ in 1998 at the University of Rome and she served as an assistant researcher at the University of 
Illinois at Urbana-Champaign (USA). She is a member of the EC Horizon 2020 advisory group for "Smart, Green and Integrated Transport" and the infrastructures representative for academia in ERTRAC.

\section{Jean-Michel Torrenti}

Jean Michel Torrenti is the $R \& D$ director of the Materials and Structures Department of IFSTTAR. He is also professor at Ecole Nationale des Ponts et Chaussées. His research concerns mechanics of concrete and its coupling with durability aspects: behavior of concrete at early age, creep, leaching. It is applied to model the behavior of structures such as bridges, nuclear power plants and nuclear waste storage. He is the co-author of several books concerning concrete and concrete structures.

\section{Bernard Jacob}

Bernard Jacob, chair of the Programme Committee of TRA2014, is deputy scientific director for transport, infrastructures and safety with IFSTTAR. His research works are in bridge and road safety, traffic loads on bridges, heavy vehicles and weigh-in-motion. He has coordinated a number of European and International research projects. He is an active member in several scientific and technical committees (OECD/ITF, PIARC, TRB, etc.) and provided expertise to the European Commission. He is professor at Ecole Nationale des Travaux Publics de l'Etat and the president of the International Society for WIM (ISWIM). He has published more than 100 scientific papers and edited 10 published volumes of international projects and conference proceedings. 


\section{Introduction}

The infrastructures of the future will have to be sustainable, seamless, resilient and durable, will respect the principles of circular economy and will have to be easy to monitor and manage. New technologies are currently available or under development to reduce the carbon footprint of infrastructures and to increase the overall sustainability and recyclability of transport while maintaining the utility and value of the infrastructures. However, the impact of these new solutions will only be effective once these are thoroughly disseminated and extensively deployed.

This volume presents a series of the most promising solutions and aims at disseminating them to improve the performances and efficiency of materials and infrastructures, through a choice of updated papers from the TRA2014 Conference. Selection is primarily based on a quality criterion, also taking into account the geographical diversity of papers in order to restore the originality and richness of current research.

\section{I.1. Main findings}

The papers contained in this volume demonstrate how technological solutions and new design and management methodologies can be implemented in different surface transport modes (roads, railways and waterways) to increase transport sustainability by improving infrastructures design, maintenance, recyclability and management. Both theoretical research and practical case studies explore topics such as characterization of pavements, bridges and soils, use of recycled and warm mix asphalts as well as high-performance materials to increase durability or to reduce the noise impact. 
New management techniques for improving infrastructure resilience both roads and railways is a very timely topic that has been selected by the European Commission and the U.S. Department of Transportation as the subject of further Euro-American cooperation. This topic is extensively covered in this volume for a number of different transport modes.

Road infrastructures are typically "low technology" structures but timely, costeffective and seamless monitoring is essential for the implementation of effective maintenance and management concepts. New solutions for pavement and soil characterization are being developed by implementing seamless technologies. These range from well-established techniques, such as ground penetrating radars (GPR) and weigh-in-motion (WIM) techniques, to innovative radar remote sensing techniques.

The development of new pavement materials is always a key topic for road and airport engineers and the implementation of recycled materials and warm mix asphalt will be the standard solution of the future. However, there is still a strong need for understanding the long-term performance of these materials in situ and for developing performance models that the designers can implement for adopting these technologies. This volume will help the designers and road managers interested in implementing these solutions and presents different case studies that will make the potential users feel more confident.

It is interesting to observe that infrastructure performances often conflict and therefore solutions such as porous asphalt, that can be very effective for noise reduction, is more sensitive to climatic changes due to the effect of freeze-thaw cycles.

Durability and maintenance are core issues for road researchers with the final aim in mind that the road of the future will have to be "Forever Open". However, local authorities are often faced with the issue of effective day to day maintenance. Infrastructure research too often focuses on highly trafficked motorways or primary road networks; therefore, it is extremely important that a research effort be specifically devoted to develop guidelines for the maintenance and repair of low volume roads, which represent a large portion of the whole road networks.

Railway and road infrastructures issues are usually tackled as separate but the recent work conducted by the joint roadmap for cross-modal transport infrastructure innovation toward a performing infrastructure has recently shown that a number of infrastructure research issues are cross-modal and therefore lessons can be learned across modes. This is clearly shown in this volume in which resilience to climatic changes covers both roads and railways and integrated modes are needed to achieve a truly resilient transport system. 
This volume will be of interest not only for the research community and in higher education but also for professionals in the area of infrastructure design and management as well as economic and institutional decision makers. They will find state-of-the-art studies of key research issues, new advanced methods and illustrative case studies.

Volume 5 of the Research for Innovative Transports set is divided into two subvolumes containing three parts each: five parts focus on roads but cover potentially cross-modal topics dealing with materials for infrastructures, auscultation and monitoring, durability and maintenance repair, recycling and sustainability issues and climate resilient roads. One part is specifically devoted to railways and inland navigation.

Sub-volume 1 contains parts 1-3. Part 1 deals with geotechnical issues and pavement materials' characterization. In this part researchers and practitioners can find new test methods and materials characterization techniques for nonconventional materials including recycled asphalt mixtures, warm mix asphalts but also fiber reinforced concrete materials.

Part 2 presents novel and high-tech solutions to monitor and assess pavement conditions to assist road authorities in this key management activity. These techniques include 3D mapping, remote sensing, GPR evaluation of pavement structural capacity and WIM monitoring solutions. The reader will also find a highly specialized study on integrating the electrical supply cables for public transport, for creating an electromagnetic induction field, in a prefabricated concrete slab.

Part 3 deals with the key road management issues of durability and maintenance repair. The recurrent theme of noise reduction has been tackled and designers and road authorities will be able to consider and compare the effectiveness of different solutions including non-conventional materials. Attention is also paid to noise issues in non-conventional analysis locations as level intersections in urban and rural areas. A very important issue for road managers is pothole repair. The guidelines developed in the POTHOLE project will be extremely helpful for local authorities looking for effective maintenance solutions.

Sub-volume 2 contains parts $4-6$. Part 4 addresses recycling and sustainability issues, presenting case studies and full-scale tests. Asphalt recycling is a core issue for reducing the carbon footprint of transportation infrastructure. Road administrations and designers will find a very interesting overview of three transnational research projects on this topic as well as a case study from Slovenia.

Part 5 analyzes railways and inland navigation issues. New concepts for low maintenance and resilient infrastructure as well as optimizing operation and 
intermodal integration within the global transport system are proposed for technicians dealing with resilient infrastructure in any transport mode. Highly specialized railway experts will find studies on clip stiffness and on new innovative solutions for transition zones between the "normal" open tracks and "rigid" track sections. Waterways researchers will find an interesting new management approach to deal with suspended sediments.

Part 6 focuses on a key infrastructure issue of the future: resilience to extreme climatic conditions. Input from three continents (Australia, Europe and North America) highlight that this global issue needs trans-national solutions. An interesting overview of two transnational projects (RIMAROCC and SWAMP) introduces the topic followed by specific solutions adopted by single countries. The effect of climatic changes on pavements is assessed to answer questions of specialized pavement engineers.

\section{I.2. Conclusions}

This volume provides an insight on research, best practices and transport policies with a focus on state-of the-art advances in the fields of infrastructures and materials. The progress made in the implementation of new materials in pavement design as well as the evolution in the process of data collection and assessment, modeling and management, assisting academics, transport professionals, practitioners and decision makers to a better understanding of the current and future trends are demonstrated.

Future infrastructure monitoring techniques will be seamless, and this volume shows that there is a significant shift of the research world in this direction. These solutions now need to become current practices to really improve the transport system.

Reducing the infrastructure carbon footprint and increasing its resilience is possible but road managers and designers need to have design and management tools as well as case studies that will allow them to gain more confidence in the adoption of new and less impacting solutions. 